\title{
Einleitung
}

Als Vorläufer der vom Institut für hellenistisch-römische Philosophie geplanten Gesamtausgabe der Schriften Epikurs und seiner Schule wird hier der zweite der drei Lehrbriefe, die bei Diogenes Laertius ${ }^{1}$ ) in der Biographie des Epikur überliefert sind, vorgelegt. Er ist an Pythokles ${ }^{2}$ ), einen jungen Anhänger, gerichtet. Für ihn soll er einen Auszug über die Himmelserscheinungen geben aus dem großen, 37 Bücher umfassenden Hauptwerk Epikurs, $\pi \varepsilon \varrho i ~ \varphi v ́ \sigma \varepsilon \omega \varsigma^{3}{ }^{3}$. Dieser Brief umfaßt die Einleitung ( $\$ 84 / 85)$, Darlegung des Zweckes: durch natürliche Erklärung aller Himmelserscheinungen den Menschen Furchtlosigkeit und Ruhe des Gemütes zu geben ( $\$ 85 / 86)$. Im Gegensatz zu der Gesamtauffassung Epikurs vom Kosmos, die ganz einheitlich sei, sollen für die einzelnen Himmelserscheinungen eine ganze Anzahl von Erklärungen zugelassen werden, da sie alle mit der Wahrnehmung übereinstimmten ( $(86 / 87)$. Es folgt eine kurze Darstellung der Entstehung der Welten (§88-90). Dann werden Erklärungen gegeben über die Größe der Himmetskörper ( $(91)$, ihre Auf- und Untergänge und täglichen Bewegungen (§92), Sonnen- und Mondwenden ( $(93)$, Voll- und Neumond (§94), das Licht des Mondes und sein Gesicht ( $(95)$, Sonnen- und Mondfinsternisse (§96), Ordnung des Umlaufs ( $\$ 97)$, Länge von Tag und Nacht, Wetterzeichen (§98). Daran schließt sich die Erklärung der atmosphärischen Erscheinungen: Wolkenbildung (§99), Donner (§ 100), Blitze ( $(101-103)$, Wirbelsturm (§ 104), Erdbeben (§ 105), Hagel (§ 106), Schnee (§ 107), Tau (§ 108), Reif, Eis (§ 109); Regenbogen und seine runde Form, Mondhof (§ 109-111). Es folgen Darlegungen über Kometen (§111), Fixsterne (§ 112), Planeten und ihre Bewegung (§ 113, 114), Sternschnuppen, nochmals Wetterzeichen (§ 115) und Schlußwort (§ 116). Die Echtheit der Lehre des Epikur in diesem Brief ist unbestritten, doch ist Epikur als Verfasser dieses Auszuges schon im Altertum in $\left.\mathrm{Z}_{\text {weifel gezogen worden }}{ }^{4}\right)$. Schon die oben gegebene Kapitelfolge zeigt die Berechtigung dieses Zweifels. UsENER ${ }^{5}$ ) führt dazu noch weitere Gründe an. Er weist auf die unverbundene Aneinanderreihung der einzelnen Naturerscheinungen hin, die allerdings durch den Stoff selbst nahegelegt wird. Aber die ständige Wiederbolung nach den Einzelerklärun-

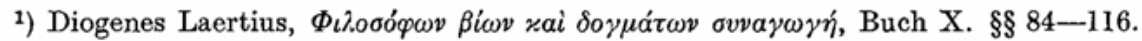

2) vgl. S. 1 b.

3) Diogenes Laertius X 26 .

4) Philodemus in den Volumina Herculanensia, coll. alt. I. Bl. 152: vं $\pi \circ \psi[i \alpha] v \tau \iota v[\dot{a}$

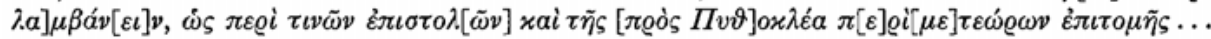

5) USENER, Epicurea, praef. XXXVII. Für die Echtheit setzt sich ein H. v. ARNIM in Epikuros, RE VI (1907) Sp. 138; neuerdings auch u. a. O. REGENBOGEN, Lukrez, Seine Gestalt in seinem Gedicht, in >Neue Wege zur Antike< II R. Heft 1 (1932) S. 61, 1. 
gen, es gebe noch viele andere, die auch möglich wären ${ }^{1}$ ), läßt vermuten, daß ein Schüler, nicht Epikur selbst, diesen Brief zusammengestellt hat, ebenso wie die oft hinzugefügte Wendung, sich immer an die Erscheinungen zu halten ${ }^{2}$ ) und dem Mythos keinen Raum zu lassen ${ }^{3}$ ), auch sich nicht um die Meinungen der Astronomen ${ }^{4}$ ) zu kümmern. Da $\Pi \varepsilon \varrho i ~ p v ́ \sigma \varepsilon \omega \varsigma$ selbst die Quelle ist, kann die Ähnlichkeit von Wortschatz und Stil im Hauptwerk und in der Zusammenfassung nicht als Beweis für die Echtheit gelten ${ }^{5}$ ). Um so mehr fällt es ins Gewicht, wenn ein bei Epikur sonst nicht nachweisbarer Ausdruck sich nur in diesem Briefe findet ${ }^{6}$ ).

Besonders auffallend ist die Behandlung der Sterne nicht an der Stelle, an der sie zu erwarten gewesen wäre, im Anschluß an $§ 98$, sondern nach den atmosphärischen Erscheinungen, zu denen Kometen und Sternschnuppen noch gezählt werden konnten ${ }^{7}$, nicht aber Fixsterne und Planeten. Dazu kommt die doppelte Aufführung der Wetterzeichen in $\$ \S 98$ und 116. Es hat fast den Anschein, als ob diese $\S \S 112-115$ nach $\S 98$ ausgefallen seien, und die beiden Möglichkeiten der Wettervoraussagen nach Erscheinungen an den Tieren und in ihrem Verhalten, die hintereinander stehen sollten, nur zufällig auseinandergerissen und dann mit fast den gleichen einleitenden Worten an verschiedenen Stellen eingesetzt worden wären. Selbst wenn das Hauptwerk, dessen Abfassungszeit sich über viele Jahre erstreckte, zusammengehörende Erscheinungen an getrennten Stellen gebracht hätte, ist eine solche Behandlung in dem kurzen Auszug Epikur selbst nicht zuzutrauen.

\section{Die Handschriften}

Die Überlieferung des Textes ist schlecht. Alle noch vorhandenen Hss des Diogenes Laertius gehen auf einen schon lücken- und fehlerreichen Archetypus zurück, - vermutlich einen Constantinopolitanus des 9. Jahrhunderts-, das zeigen die gemeinsamen Lücken und Fehler des Textes. Da allen Bemühungen, Einblick in die Handschriften selbst zu nehmen, der Erfolg versagt wurde, werden die Hss und der kritische Apparat hier nach den wichtigsten Ausgaben, vor allem der vON DER MüHLLS, gegeben.

Die älteste Hs, die auch im Vergleich mit den andern noch den besten Text gibt,

$\mathrm{B}$ ist $\mathrm{B}=$ Borbonicus Neapolitanus gr. III B 29 aus dem 12./13. Jh. Ihr .

$\mathrm{P}$ steht $\mathrm{P}=$ Parisinus gr. 1759 aus dem 14. Jh. nahe, doch haben hier drei Korrektoren Abänderungen eingetragen, so daß die Lesarten der ersten

1) $\S 86 ; 87 ; 94 ; 95 ; 97 ; 98 ; 100 ; 102 ; 104 ; 108 ; 113 ; 115$.

2) $\S 91 ; 93 ; 96 ; 97 ; 99 ; 102$.

3) $\S 87 ; 104 ; 115$.

4) $\S 93 ; 112$.

5) vgl. PreHn, De Epicuro ad Pythoclem epistula. Diss. Greifsw. 1925.

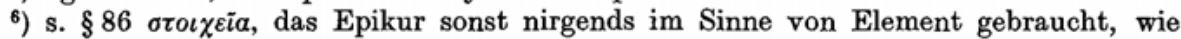

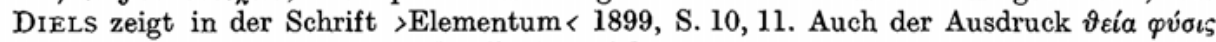
in $\S \S 97,113,115$ kommt sonst bei Epikur nicht vor.

$\left.{ }^{7}\right)$ s. ACHILles, Isagoga Excerpta 32 in Commentariorum in Aratum reliquiae, hrsg. von E. MAASS (1898) S. 68. 
Co Hand nicbt immer erkennbar sind. Da sind von Nutzen Co $=$ Constantinopolitanus

$\mathrm{Q}$ Veteris Serail aus dem 14./15. Jh. und $\mathrm{Q}=$ Parisinus gr. 1758

$\mathrm{W}$ aus dem 14./15. Jh. sowie $\mathrm{W}=$ Vaticanus gr. 140 aus dem 14. J., da sie aus P abgeschrieben wurden, ehe die Verbesserungen vorgenommen worden waren. $\mathrm{P}^{2}$ gehört noch dieser ersten Hss-Klasse an, $\mathrm{P}^{3}$ dagegen bringt Lesarten der zweiten, weniger guten Klasse. Der älteste Vertreter dieser

F zweiten Klasse ist $\mathbf{F}=$ Laurentianus gr. 69, 13 aus dem 13. Jh.

$\mathrm{Z} \quad \mathrm{Z}=$ Lobcowicensis Raudnitzianus, in der von $\mathrm{Z}^{3}$ abgeänderten Form ist die

f Quelle für f = Erstausgabe des Matthaeus Aurigallus, die 1533 bei Froben in Basel erschien.

T Ebenfalls zu dieser zweiten Klasse gehören noch $\mathrm{T}=$ Urbinas gr. 109 und

D $\mathrm{D}=$ Borbonicus gr. III B 28, die wohl dem 15. Jh. angehören, und

G $\mathrm{G}=$ Laurentianus 69,28 und $\mathrm{H}=$ Laurentianus 69,35 , die aus dem 14. Jh.

$\mathrm{H}$ stammen.

\section{Nebenüberlieferung}

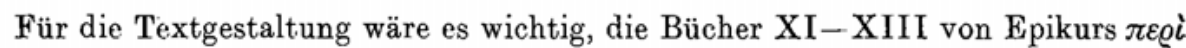
qv́$\sigma \varepsilon \omega \varsigma$ heranziehen zu können. Das Werk ist wahrscheinlich schon im späten Altertum verlorengegangen, und die Reste, die die in Herculaneum aufgefundenen, sehr beschädigten und fast zu Kohle gewordenen Papyrusrollen geliefert haben, sind nicht sehr ergiebig. A. Vogliano gibt in seiner Sonderausgabe von Buch $\mathrm{XI}^{1}$ ) eine genaue Übersicht über die vorhergehenden Publikationen und einen - soweit es möglich ist - geordneten und ergänzten Text der zerstreuten Reste. Da diese Fragmente aber einzelne Teilgebiete der Naturlehre sehr ausführlich behandeln, können sie für die kurze Zusammenfassung, wie sie der Brief an Pythokles darstellt, nicht viel helfen.

Mehr Bedeutung für den Text haben daher die Doxographen ${ }^{2}$ ), die deshalb auch, nach USENERS Vorbild, im Parallelenapparat angeführt werden.

Leider sind die beiden Werke nicht erhalten, die Epikur selbst vielleicht benutzt

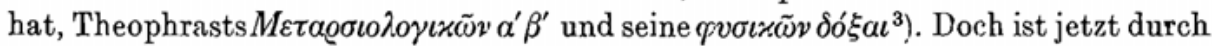
ein arabisches Fragment ein kleiner Teil eines dieser Werke etwas besser bekannt geworden ${ }^{4}$ ). G. BERGSTRÄSSER hat aus einer Konstantinopeler Handschrift vom Jahre 1446/475) eine Abhandlung Theophrasts, die Al-Hasan ibn Bahlûl aus dem Syrischen ins Arabische übersetzt hat ${ }^{6}$ ), herausgegeben. Ihre Bedeutung gerade auch für den Brief an Pythokles legt E. Reitzenstein dar ${ }^{7}$ ). Daher werden auch die Paragraphen dieses Fragments im Parallelenapparat mit angeführt.

1) Achille Vogliano, I resti dell XI libro del $\Pi \varepsilon \varrho i$ pv́ $\sigma \varepsilon \omega \varsigma$ di Epicuro in den Publications de la société Fouad I. de papyrologie, Textes et Documents. 1940.

$\left.{ }^{2}\right)$ Doxographi Graeci, coll. Hermann Diels 23929.

3) O. Regenbogen, Theophrastos von Eresos, RE, Suppl. Bd. VII 1353ff.

4) Ebd. 1408-1410.

5) Ms Ašir Efendi I, 1164 .

6) G. BergStrÄSSER, Neue meteorologische Fragmente des Theophrast, arabisch und deutsch, in den Stzber. Heid. Ak. d. Wiss. Phil. Hist. Kl. 1918, Nr. 9; s. bes. S. 13.

${ }^{7}$ ERICH REITZENSTEIN, Theophrast bei Epikur und Lukrez, in: Orient und Antike 2, 1924. S. auch H. Sт Rонм, Zur Meteorologie des Theophrast, Philologus 92, 1937, $268 \mathrm{ff}$ u. $403 \mathrm{ff}$; und Theophrast und Poseidonios, Hermes 81, 1953, $278 \mathrm{ff}$. 
Besonders wichtig ist Lukrez, der in seinem Lehrgedicht $>$ De rerum natura $<{ }^{1}$ ) viel aus Epikurs Hauptwerk erhalten hat, obgleich nicht feststeht, ob er es selbst benützt hat ${ }^{2}$ ), oder ob er es aus den Schriften jüngerer Epikureer kannte ${ }^{3}$ ). Er gibt zu den einzelnen Himmelserscheinungen meist mehr Erklärungen, als sie der Pythoklesbrief bietet. Die Verse seines Gedichtes sind auch in die Belegstellen mit aufgenommen.

\section{Ausgaben}

Da eine Epikur-Bibliographie in diesen Veröffentlichungen demnächst herauskommen wird, werden hier nur kurz die Ausgaben aufgeführt, die bei der Aufstellung des kritischen Apparates durch ihre Verbesserungsvorschläge wichtig sind.

Es sind dies außer der schon genannten Erstausgabe die Ausgaben des Diogenes Laertius von

$\begin{array}{llll}\text { Johannes Sambucus } & 1566 & \text { Marcus Meibomius } & 1694 \\ \text { Heinrich Stephanus } & 1577 & \text { Heinr. Gust. Huebner } & 1828 \\ \text { Isaac Casaubonus } & 1583 & \text { Carl Gabriel Cobet } & 1862 \\ \text { Thomas Aldobrandinus } & 1594 & \text { Robert Drew Hicks } & 1925 \\ \text { Aegidius Menagius } & \mathbf{1 6 6 4} & & \end{array}$

Die älteren Ausgaben, die meist nur wenige Handschriften und oft die minder guten zugrunde legten, haben jetzt ihre Bedeutung hauptsächlich dadurch, daß die Herausgeber zu dem schwierigen und fehlerhaft überlieferten Text manche Konjekturen gebracht haben, die auch jetzt nützlich sind.

Wichtiger sind die Ausgaben, die nur das 10. Buch des Diogenes Laertius oder Teile daraus meist mit Erklärungen umfassen. PETER GASSENDI hat mit seiner Ausgabe des X. Buches des Diogenes Laertius und seinem Kommentar 1647 und 1649 (vgl. jetzt Opera omnia, Bd. V, 1658) die Atomlehre Epikurs zu neuem Leben erweckt.

JoH. GotTlieb SchneIder gibt in seinen Physica et Meteorologica 1813 nur die Lehrbriefe I und II mit Anmerkungen. Grundlegend war die Sammlung alles von Epikur noch Erfaßbaren, Lehrbriefen und Fragmenten, durch HERMANN USENER in den Epicurea, 1887. Er hat dem Text des Diogenes Laertius durch die Heranziehung und Benutzung aller Handschriften erst einen kritischen Apparat gegeben. PETER VON DER MÜHLL hat für seine Ausgabe Epicuri Epistulae Tres et Ratae Sententiae, 1922, die Handschriften alle, zumeist in Original, kollationiert, darnach den kri-

1) T. LUCRETIUS CA RUS, De rerum natura. Benutzt wurde die Ausgabevon H. Diels, 1923.

2) J. Woltjer, Lucretii philosophia cum fontibus comparata, Groningen 1877. Vgl. auch E. REITZENSTEIN a. a. O.

3) Usener, Epicurea, praef. XXXVI; Diels, Elementum 1899, S. 9; 12; Diels, Lukrezstudien, Berl. Stzber. 1920, S. 17; Lück, Die Quellenfrage im 5. und 6. Buch des Lukrez, Diss. Breslau 1932, und die Rezension dazu von E. Reitzenstein im Gnomon 1933, S. $542 \mathrm{ff}$. 
tischen Apparat aufgebaut und den schwierigen Text so getreu wie möglich wiedergegeben, indem er auch die Stellen, die ibm spätere Hinzufügungen zu sein scheinen, doch in den Text, nur in kleinerem Satz und in Klammern, aufnimmt.

Auf seinen kritischen Apparat stïtzen sich auch die beiden neuesten Ausgaben, die von R. D. Hicks in seiner Edition des Diogenes Laertius in der Loeb Classical Library ,1925, und die von CYRIL BAILEY, Epicurus, The extant remains, Oxford 1926.

\section{Übersetzungen}

Wie bei den Ausgaben werden auch hier nur die zur Textherstellung herangezogenen neuren Übersetzungen angeführt.

A. Kochalsky, Das Leben und die Lehre Epikurs, Diogenes Laertius Buch X, 1914, das außer der Übersetzung in einem Anhang kritische Anmerkungen zum griechischen Text bringt.

E. Bignone, Epicuro. Opere, frammenti, testimonianze, 1920, der seiner italienischen Übersetzung wichtige Verbesserungen des griechischen Textes beifügt.

0. APELT, Diogenes Laertius, der seiner Übersetzung Erläuterungen beigibt, 1921. (Meiner's Philosoph. Bibliothek 53 u. 54).

R. I. HICKs, der die englische Übersetzung für die Ausgabe des Diogenes Laertius in der Loeb-Library besorgte, 1925.

A. ERnout-L. Robin haben zu Lucrèce, De rerum natura, im Commentaire exégétique et critique $\mathrm{Bd}$. I die französische Übersetzung von ERNOUT der drei

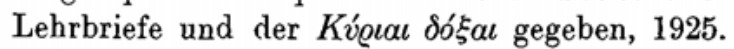

C. BAILEY hat seiner Ausgabe des griechischen Textes die englische Version gegenübergestellt und Text und Änderungen in Anmerkungen erläutert, 1926.

O. GIGON, Epikur. Von der Überwindung der Furcht. Katechismus, Lehrbriefe, Spruchsammlung, Fragmente, 1949, gibt eine sehr gut lesbare deutsche Übersetzung, die die langen Sätze des Originals in passenden Teilungen wiedergibt.

Außer den im Vorwort genannten sind noch folgende Werke öfters herangezogen worden:

E. Bignone, L' Aristotele Perduto, I. II. o. J.

O. GILBERT, Die meteorologischen Theorien des griechischen Altertums, 1907.

M. HeEger, De Theophrasti qui fertur $\pi \varepsilon \varrho i ~ \sigma \eta \mu \varepsilon i ́ \omega v$ libro, Diss. Leipzig 1889.

J. ROEHR, Beiträge zur antiken Astrometeorologie, im Philologus 83 (1928) S. 259 ff.

K. WESSELY, Bruchstücke einer antiken Schrift über Wetterzeichen. Wiener Stzber. 142, 1900.

H. Widmann, Beiträge zur Syntax Epikurs, in den Tübinger Beiträgen zur Altertumswissenschaft 24, 1935.

Höchst wichtig für die Textgestaltung waren

R. Fhilippson, Recension der Ausgabe v. d. Mühlls in B. Ph. W. 43 (1923) $1092-.1100$

und vor allem A. BARIgazzI, Note critiche alla lettera a Pitocle di Epicuro, in den Studi Italiani di Filologia Classica 23 (1949) 179-213, der den überlieferten Text, soweit es nur irgend möglich ist, verteidigt und erklärt. 